\title{
Precisamos realmente de educação para os meios?
}

David Buckingham

Professor de Mídia e Comunicações na Escola de Ciências Sociais da Universidade Loughborough. Até 2012, foi professor de Educação no Instituto de Educação da Universidade de Londres, onde dirigiu o Centre for the Study of Children, Youth and Media. Sua pesquisa é voltada à Educação para os Meios (media education) e às interações de crianças e jovens com a midia. É autor ou coautor de 25 livros relacionados a esses temas.

Resumo: $\mathrm{O}$ artigo traça um breve histórico do desenvolvimento da Educação para os Meios no Reino Unido, relacionando-o à evolução global do conceito e suas aplicações, além de realizar análise detalhada do fenômeno afeito à Mídia 2.0 - refletindo sobre seus impactos, benefícios e utilizações na mídia educação ao redor do mundo.

Palavras-chave: Mídia-educação; web 2.0; comunicação e educação; mídia digital.
Abstract: The article describes briefly the history of Media Education in the United Kingdom, relating it to the global evolution of the concept and its application, in order to undertake a detailed analysis of this phenomenon in relation to Media 2.0 - reflecting on its impacts on Media Education all over the world.

Keywords: Media education; web 2.0; communication and education; digital media.

\section{O ENSINO DE MÍDIA NA ERA DA CULTURA PARTICIPATIVA ${ }^{1}$}

O advento da mídia digital, na visão de muitos analistas, requer novos paradigmas dentro do mundo acadêmico, na prática criativa e na pedagogia. Segundo alguns, os "novos" meios de comunicação de massa são tão fundamentalmente diferentes dos "antigos", que chegam a necessitar, também, de diferentes métodos de análise, de estruturas teóricas e conceituais e de engajamento político e intelectual. Em relação à educação, acredita-se que as possibilidades interativas e participativas da mídia digital transcendam as limitações da mídia "de massa", que é hierárquica e vai de cima para baixo; e que, portanto, essas possibilidades poderiam enfraquecer a autoritária "política do conhecimento", como é chamada na pedagogia tradicional. O potencial que a mídia digital oferece aos estudantes para que se tornem criadores - ao invés de "consumidores" do conhecimento - é considerado por alguns como algo quase revolucionário.

Neste texto, tentarei questionar algumas dessas asserções e avaliar suas implicações na prática educacional. Minha preocupação específica se relaciona à prática da Educação para os Meios: interesso-me mais sobre o quê e como devemos ensinar a respeito da mídia do que sobre o uso da mídia como material de ensino ou recurso didático (isto é, a mídia educacional). Meu foco

1. Publicado originalmente em DROTNER, Kirsten; SCHRODER, Kim. Digital Content Creation [Criação de conteúdo digital]. New York: Peter Lang, 2010. 
comunicação \& educação • Ano XVII • número 2 • jul/dez 2012

2. BUCKINGHAM, David. Media education: Literacy, learning and contemporary culture [Educação para os meios: literacia, aprendizado e cultura contemporânea] Cambridge: Polity, 2003; BURN, Andrew; DURRAN James. Digital anatomies: analysis as production in media education [Anatomias digitais: a análise como produção em educação para os meios]. In: BUCKINGHAM, David WILLETT, Rebekah (eds.) Digital generations: Children, young people and new media [Gerações digitais: crianças, jovens e novos meios de comunicação de massa]. Mahwah, NJ: Erlbaum, 2006. p. 273-294

3. Revisões desse trabalho podem ser encontradas em: BUCKINGHAM, David. Media education: Literacy, learning and contemporary culture [Educação para os Meios: literacia, aprendizado cultura contemporânea]. Cambridge: Polity, 2003 BUCKINGHAM, David; BANAJI, Shakuntala; BURN Andrew; CARR, Diane CRANMER, Susan; WILLETT, Rebekah. The media literacy of children and young people: A review of the academic research [Literacia mediática de crianças e jovens: uma análise da pesquisa acadêmica] Journal of Educational Media and Hypermedia, 16 , p. 323-349. London: Ofcom, 2005. Exemplos mais recentes encontram se em edições especiais de Learning, Media and Technology (2007) e Media International Australia (2006).

4. Parte desse debate pode ser acessado em <http://twopointzeroforum.blogspot.com/>. O blog de Merrin pode se consultado em: <http:// mediastudies2point0.blogspot.com/> enquanto trabalho de Gauntlett est disponivel no site: <http:// www.theory.org.uk $>$ (acessos em: mar. 2009). concentra-se no ensino escolar, embora também possa ter implicações para a prática da Educação para os Meios nas universidades.

A Educação para os Meios tem uma longa história no Reino Unido, datando de mais de sessenta anos. Temos cursos de estudos de mídia, validados por especialistas, que podem ser frequentados, na maioria das escolas, por alunos de 14 anos ou mais; além disso, a Educação para os Meios é uma dimensão do ensino da língua materna (da matéria "Inglês") ao longo do ensino secundário. Embora seja menos uniforme a abordagem do tema nas escolas primárias, nos últimos tempos os formuladores de políticas educacionais reconhecem, cada vez mais, que todas as crianças devem aprender sobre a mídia como parte fundamental do ensino e, em particular, da capacidade de avaliar informações ${ }^{2}$. Sendo assim, falo a partir de um contexto onde já existe um elemento bem estabelecido de prática educacional: a Educação para os Meios no Reino Unido é orientada por uma estrutura conceitual bem coerente e há uma boa noção do que "funciona" na sala de aula. O que é muito importante em relação ao foco deste texto é que a Educação para os Meios nas escolas do Reino Unido sempre foi relacionada à criatividade e à crítica: não apenas sobre como fazer mídia, mas também sobre como analisar a mídia.

Evidentemente isto não quer dizer que possamos dispensar os debates, ou pensar que não há áreas carentes de desenvolvimento. Na realidade, há um crescente impulso de pesquisa que está focalizando algumas das contradições e limitações da prática estabelecida na Educação para os Meios ${ }^{3}$. Sob esse aspecto, grande parte do debate gira em torno de questões fundamentais sobre o conhecimento e a aprendizagem: o que nós achamos que os jovens já sabem sobre comunicação, o que eles precisam saber, e como eles deveriam aprender? $\mathrm{O}$ advento da mídia digital tem algumas implicações interessantes a respeito dessas questões. Como veremos, alguns dizem que as mudanças contemporâneas no meio mais amplo da comunicação exigem uma reconsideração dos objetivos e métodos fundamentais da Educação para os Meios - não apenas relativa ao conteúdo do currículo, mas também à nossa pedagogia e aos nossos métodos de ensino. Mas seriam essas mudanças tão fundamentais quanto seus defensores sugerem? E é realmente imprescindível que a era da "Mídia 2.0" também requeira a "Educação para os Meios 2.0"?

\section{ESTUDOS DE MÍDIA 2.0 E A REINVENÇÃO TOTAL DE TUDO}

No começo de 2007, houve um breve, porém intenso, debate nos murais acadêmicos dos Estudos de Mídia do Reino Unido, focalizando a noção de "Estudos de Mídia 2.0". Esse debate foi iniciado por William Merrin, da Universidade de Wales, em Swansea, e prontamente abraçado por David Gauntlett, da Universidade de Westminster. Subsequentemente, juntaram-se vários outros participantes, alguns conhecidos e outros anônimos - assim como grupos de professores de Estudos de Mídia em escolas e faculdades, fazendo seus comentários on-line ${ }^{4}$. A 
discussão girou, principalmente, em torno de como a disciplina acadêmica de Estudos de Mídia (e Estudos Culturais) era ensinada nas universidades, embora eu não vá abordá-la aqui ${ }^{5}$. Meu interesse se concentra nas implicações para os professores nas escolas; e, apesar do tom às vezes polêmico e um tanto irritante desse debate, ele levantou algumas questões importantes que são, na verdade, ainda mais pertinentes para professores de crianças e jovens.

Muitos adolescentes hoje em dia estão crescendo com o conjunto de comunicações participativas coletivamente conhecidas com "Web 2.0" - que se constituem em usar redes sociais e jogos on-line, compartilhar fotos e vídeos, escrever em blogs, publicar em podcasting, remixagens, mashups, wikis, machinima e utilizar conteúdo gerado pelos usuários, entre outras atividades. Estes novos meios de comunicação não substituíram os antigos: em média, os jovens ainda passam muito mais tempo diante da televisão do que on-line ${ }^{6}$, e muitos deles ainda continuam, obstinadamente, a ler livros. No entanto, se basearmos nosso ensino em formas de comunicação que, se não forem totalmente antiquadas, no mínimo apresentam somente uma parte do ambiente que os jovens estão vivenciando no momento, existe o claro perigo de que o que estamos fazendo em sala de aula venha a se tornar irrelevante em suas vidas. Meu argumento é que isto não é simplesmente uma questão de conteúdo do currículo - de ensinar aos jovens a maneira de analisar páginas da internet ou anúncios de televisão, por exemplo. Entusiastas dos novos meios de comunicação tipicamente alegam que as novas mídias exigem uma orientação completamente diferente no tocante à informação, além de outra fenomenologia de uso, outra política de conhecimento e um modo de aprendizagem diferente. Se for assim, isso potencialmente tem implicações de longo alcance para a pedagogia - e não somente sobre o que nós ensinamos, mas também sobre como ensinamos.

Para William Merrin (2008), os novos meios de comunicação de massa representam um desafio fundamental ao nosso direito de ensinar:

Nosso temor da tecnologia frequentemente inclui o nosso próprio uso pessoal dela. Na era da transmissão por rádio e televisão, compreendíamos, de modo geral, os princípios técnicos básicos dos meios de comunicação dominantes e entendíamos o seu uso, compartilhando-o com nossos alunos. No entanto, hoje em dia, os palestrantes estão ficando para trás em seus conhecimentos sobre quais tecnologias estão à disposição, sobre suas possibilidades técnicas e até mesmo sobre como elas funcionam, como usá-las e para que fins estão sendo usadas. Como já foi dito, não compartilhamos mais uma cultura em comum com nossos alunos. A menos que possamos nos manter atualizados sobre essas tecnologias que estão em constante mudança e sobre seus usos, e a não ser que elas se tornem parte importante de nossas vidas como o são para nossos alunos, corremos o risco de perder não só a habilidade de ensiná-las, mas até mesmo o direito de ensiná-las. Na época em que assistíamos à televisão e a estudávamos, tínhamos o direito de ensiná-la. No futuro, se não estivermos baixando conteúdos ou documentos da internet, compartilhando, ripando, queimando, enviando mensagens, fazendo networking, jogando, montando e produzindo, acabaremos por perder esse direito ${ }^{7}$.
5. Algumas discussões interessantes sobre o tema podem ser encontradas em um trabalho especial do International Journal of Cultural Studies 5(4), 2002, e em: BLACKMAN, Shane. "Decanonised knowledge" and the radical project: towards an understanding of cultural studies in British universities. ["Conhecimento descanonizado" e o projeto radical: para entender os estudos culturais nas universidades britânicas]. Pedagogy, Culture and Society, 8, p. 43-67, 2000. 6. OFCOM. Media Literacy: Report on UK Children's Media Literacy [Literacia mediática: relatório sobre a literacia mediática das crianças do Reino Unido]. London: Ofcom, 2008.

7. MERRIN, Willian. Media Studies 2.0 [Estudos de Mídia 2.0]. Disponível em: <http://twopointzeroforum.blogspot.com/>. Acesso em: 16 maio 2009. 
comunicação \& educação • Ano XVII • número 2 • jul/dez 2012

8. BUCKINGHAM, David. "Creative" visual methods in media research: possibilities, problems and proposals [Os métodos visuais "criativos" na pesquisa de mídia: possibilidades, problemas propostas]. Media, Culture and Society, 31(4) p. $633-652,2009$.

9. GROSSBERG, Lawrence. Cultural Studies vs. political economy: is anybody else bored with this debate? [Estudos culturais vs. economia política: há mais alguém entediado com esse debate?]. Critical Studies in Mass Communication, $12, \mathrm{p}$ 72-81, 1995.

10. GAUNTLETT, David. Wide angle: is it time for Media Studies 2.0? [Grande angular: é chegada a hora para os Estudos de Mídia 2.0?]. Media Education Association Newsletter, 5, p. 3-5 2007.
Certamente haverá questões a discutir sobre a narrativa histórica oferecida aqui - entre elas a noção de que, em determinado momento do passado, havia uma "cultura comum", uma experiência compartilhada dos meios de comunicação entre os professores (ou "palestrantes") e os alunos, a qual agora se perdeu. No entanto, a questão mais desafiadora é relativa ao "direito de ensinar" - essencialmente, a respeito da legitimidade de ensinar na era da Media 2.0.

Os argumentos desenvolvidos por Merrin e Gauntlett, em suas várias contribuições, giram em torno de uma oposição binária (bastante fora de moda, em estilo estruturalista) entre "1.0" e "2.0". Um resumo esquemático, baseado principalmente em Gauntlett (2007), ficaria mais ou menos assim:

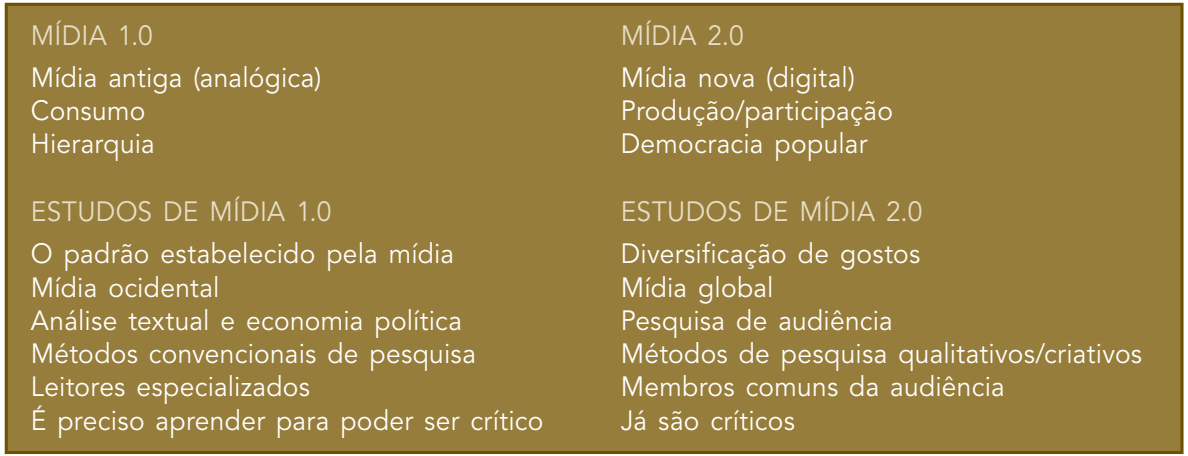

Como muitos modelos binários semelhantes, este peca pela tendência de lidar com opostos absolutos e mesclar questões bem distintas. Por exemplo, a distinção entre os meios de comunicação de massa ocidentais e os globais não se delineia na distinção entre a mídia "antiga" e a "nova" - particularmente em uma situação em que a maioria da população do Sul global nem mesmo tem acesso à eletricidade, muito menos à internet banda larga. Outros debates podem ocorrer a respeito dos métodos de pesquisa e dos méritos das chamadas abordagens "criativas" da pesquisa de audiência, as quais discuti em um outro estudo $^{8}$. Até certo ponto, Gauntlett também está retomando um velho debate acadêmico dos Estudos de Mídia entre a economia política e a pesquisa de audiência - um debate que, como Lawrence Grossberg ${ }^{9}$ e outros sugeriram, está sendo indevidamente polarizado.

Mesmo assim, há aqui questões pedagógicas interessantes que retomam a discussão a respeito da legitimidade do ensinar. Poderíamos caracterizar esta posição como uma espécie de populismo pedagógico. Gauntlett, por exemplo, desdenha a análise textual ao dizer que uma característica básica dos Estudos de Mídia 1.0 é uma "tendência a fetichizar os "especialistas", cujas interpretações de cultura popular são consideradas mais importantes do que as de outros membros do público (e valorizando "não procedimentos" de falsos especialistas, tais como os da semiótica)". Em contraste, nos Estudos de Mídia 2.0, esta abordagem é "substituída por um foco nos significados corriqueiros produzidos por um leque variado de membros do público" ${ }^{10}$. Da mesma forma, os Estudos de Mídia 1.0 são caracterizados por "uma crença de que os alunos devem aprender 
como "ler" a mídia em um estilo "crítico" apropriado". Nos Estudos de Mídia 2.0, isto já não se faz necessário:

A opinião condescendente de que os alunos devem aprender como "ler" os meios de comunicação é substituída pelo reconhecimento de que o público da mídia, em geral, já é extremamente capacitado para interpretar seu conteúdo, com olhar crítico e entendimento das suas técnicas de comunicação contemporâneas - graças, em grande parte, à enorme cobertura sobre o assunto feita pelos próprios meios de comunicação populares ${ }^{11}$.

Obviamente, aqui há certa provocação deliberada, mas há, também, questões interessantes a respeito da aprendizagem. Se as pessoas em geral já estão criando seus próprios significados variados, participando e produzindo seus próprios meios de comunicação da forma extremamente capaz e crítica que Gauntlett está sugerindo - e ele não é o único a argumentar desta maneira -, então, o que precisam aprender, e o que devemos ensinar-lhes?

\section{OS LIMITES DA MÍDIA 2.0}

Os defensores dos Estudos de Mídia 2.0 comungam o que Richard Barbrook e Andy Cameron ${ }^{12}$ chamaram, muitos anos atrás, de "a ideologia californiana" - uma espécie de libertarianismo cibernético populista que afirma que, de algum modo, as pessoas comuns ganharão poder por meio da tecnologia e que as mídias digitais serão inerentemente emancipadoras e contraculturais. Esta abordagem está certamente aparente na celebração de "criatividade" e "participação" por si mesmas, assim como na valorização das "pessoas comuns" em oposição a procedimentos críticos espúrios e atitudes condescendentes dos que se autodenominam "especialistas".

Os problemas mais amplos dessa abordagem têm sido largamente explorados (por exemplo, em Robins e Webster $^{13}$; Woolgar ${ }^{14}$ e Buckingham ${ }^{15}$ ). De forma geral, ela se baseia em um determinismo tecnológico - uma visão segundo a qual a tecnologia produz mudança social de maneira um tanto autônoma. Neste contexto, assim como em discussões mais amplas sobre educação, ela também implica a noção da "geração digital" - a ideia de que a tecnologia produziu uma mudança geracional absoluta e fundamental e de que os jovens, hoje em dia, são automaticamente hábeis em tecnologias ou letrados em mídias. Como vários críticos argumentam, esta visão representa uma espécie de essencialismo ou "exoticismo" da juventude, ignorando, em si, a diversidade e as desigualdades nas experiências dos jovens, assim como as continuidades ao longo das gerações ${ }^{16}$.

Uma crítica compreensiva da retórica da "Mídia 2.0" ficaria além do escopo deste texto, embora seja importante levantar algumas questões críticas essenciais. O termo "Web 2.0" parece ter sido criado, em 2001, pelo empreendedor de marketing digital Tim O'Reilly e seus associados ${ }^{17}$. De fato, pode-se dizer que ele reflete uma tentativa de reformular a imagem dos negócios da internet depois do estouro da chamada "bolha ponto com". O próprio O'Reilly, aparentemente, já se cansou dessa ideia; enquanto outros - inclusive Tim Berners-Lee,
11. Ibid.

12. BARBROOK, Richard; CAMERON, Andy. The Californian ideology $[A$ ideologia californiana]. Disponível em: <http:// www.hrc.wmin.ac.uk/theory-californianideology. html>. Acesso em: 8 nov. 2008.

13. ROBINS, Kevin; WEBSTER, Frank. Times of the technoculture [Época da tecnocultura]. London: Routledge, 1999.

14. WOOLGAR, Steve. Virtual society? Technology, cyberbole, reality [Sociedade virtual? Tecnologia, "cyberbole", realidade]. Oxford: Oxford University Press, 2002.

15. BUCKINGHAM, David; PINI, Maria; WILLETT, Rebekah. "Take back the tube!" The discursive construction of amateur film-and video-making ["De volta à televisão!" A construção discursiva na criação de filmes e vídeos amadores]. Journal of Media Practice, 8, p. 183-201, 2007.

16. FACER, Keri; FURLONG, Ruth. Beyond the myth of the "cyberkid": young people at the margins of the information revolution [Além do mito do "cyberkid": jovens à margem da revolução informática]. Journal of Youth Studies, 4, p. 451-469, 2001; BUCKINGHAM, David. Is there a digital generation? In: BUCKINGHAM, David; WILLETT, Rebekah (eds.). Digital generations, cit., p. 1-17; HERRING, Susan. Questioning the $c$ divide: technological exoticism and adult constructions of online youth identity [Questionando o divisor geracional: exoticismo tecnológico e construções adultas da identidade on-line do jovem]. In: BUCKINGHAM, David (ed.). Youth, identity and digital media [Juventude, identidade e mídia 
comunicação \& educação • Ano XVII • número 2 • jul/dez 2012

digital]. Cambridge: MIT Press, 2008. p. 71-92.

17. O'REILLY, Tim (2005). What is Web 2.0? Design patterns and business models for the next generation of software [O que é Web 2.0? Padrões de design e modelos de negócios para a próxima geração de software] 2005. Disponível em: <http://www.oreillynet. com/pub/a/oreilly/tim/ news/2005/09/30/whatis-web-20.html>. Acesso em: 17 mar. 2009.

18. ANDERSON, Nate Tim Berners-Lee on Web 2.0: "nobody even knows what it means" [Web 2.0: "Ninguém nem mesmo sabe o que sig nifica"]. Disponível em: Ars Technica (<http:// arstechnica.com/business/news/2006/09/7650. ars>). Acesso em: 17 mar. 2009; e ZIMMER, Michael. Critical Perspectives on Web 2.0 [Perspectivas críticas na Web 2.0]. Dis ponível em: <http://www. uic.edu/htbin/cgiwrap/ $\mathrm{bin} / \mathrm{ojs} /$ index.php/fm/is sue/view/263/showToc $>$. Acesso em: 20 ago. 2008.

19. STREETER, Thomas The cable fable revisited: discourse, policy and the making of cable television. [A fábula do cabo revista: discurso, política e a criação da televisão a cabo]. Critical Studies in Mass Communication, 4, p. 174-200, 1987.

20. BUCKINGHAM, David PINI, Maria; WILLETT, Rebekah. "Take back the tube!" The discursive construction of amateur film-and video-making ["De volta à televisão!" A construção discursiva na criação de filmes e ví deos amadores]. Journal of Media Practice, 8, p. 183-201, 2007.

21. MARVIN, Carolyn When old technologies were new [Na época em que as velhas tecnologias geralmente aceito como o inventor da World Wide Web - questionam se a Web 2.0 é mesmo realmente diferente da Web 1.0, já que a infraestrutura tecnológica básica e muitas das formas ou gêneros da Web 2.0 vêm sendo usadas desde o começo da internet ${ }^{18}$.

Promover o potencial das novas mídias e do poder que outorgam, em termos de democratização, não é nenhuma novidade. Podemos olhar retrospectivamente para os argumentos sobre a TV a cabo na década de $1970^{19}$ ou sobre o vídeo portátil na década de $1980^{20}$ - embora, de fato, a maioria das novas tecnologias das mídias tenha sido recebida com aclamações sobre seu potencial inerentemente radical ${ }^{21}$. Foi, sem dúvida, o caso dos "antigos" meios de comunicação, televisão e rádio, assim como da imprensa ${ }^{22}$ : aparentemente, todos esses meios de massa iriam outorgar poder às pessoas, subverter o controle do conhecimento por parte das elites e possibilitar aos cidadãos comuns expressarem-se e fazerem-se ouvir, criando novas formas de colaboração nas maneiras revolucionárias que, agora, são precisamente características da mídia digital. E, em cada caso, os efeitos posteriores foram muito mais complexos e equívocos do que aqueles que seus defensores proclamavam.

Uma das razões disso foi a difusão desnivelada e verdadeiramente desigual das inovações tecnológicas. No caso da "Web 2.0", as estatísticas sobre os padrões de uso não são inteiramente confiáveis. Por exemplo, em um estudo recente feito pela Fundação Pew ${ }^{23}$, 64\% dos adolescentes americanos disseram ter criado ou compartilhado "conteúdos" on-line. Muito embora este número pareça incluir os perfis nos sites de redes sociais, 39\% afirmam ter publicado trabalhos artísticos, escrita criativa, fotografias ou vídeos. Por outro lado, estatísticas fornecidas pela agência de pesquisa de mercado Hitwise sugerem que, entre os usuários do YouTube - o site mais acessível de compartilhamento de vídeo on-line somente $0,16 \%$ realmente fazem upload de material, não estando claro qual a porcentagem de material original e qual a porcentagem de clips pirateados dos meios de comunicação comerciais ${ }^{24}$. O mesmo estudo constatou que $0,2 \%$ dos que acessam o site Flick $r$ fazem upload, enquanto apenas 4,6\% dos usuários editaram ou escreveram para Wikipedia (um número que eu suspeito ser alto).

Umas das dificuldades evidentes aqui (e certamente para este volume como um todo) é definir o que queremos dizer com "criar conteúdo". Quando realizamos uma pesquisa em forma de questionário, em meados da década de 1990 - ou seja, no início da "criatividade digital" -, meu colega Julian Sefton-Green e eu descobrimos um número surpreendentemente alto de jovens que se diziam engajados em atividades criativas de multimídia em seus computadores; porém, quando os sondamos mais fundo por meio de entrevistas e visitas em domicílio, verificamos que, na realidade, isso acontecia bem pouco ${ }^{25}$. A maioria desses jovens parecia saber o que poderiam, potencialmente, fazer com a tecnologia; mas, também à maioria deles, faltava a motivação pessoal e social para realmente fazê-lo. Ainda hoje em dia, eu diria que somente uma proporção muito pequena de usuários está, de fato, gerando conteúdo original: a maior parte está simplesmente "consumindo" conteúdo, como sempre fez. 
Precisamos realmente de educação para os meios? - David Buckingham

Além do mais, corre-se o risco aqui de representar incorretamente o que a maioria das pessoas está realmente fazendo, quando se declara "criando conteúdo". Jean Burgess ${ }^{26}$ criticou, com razão, os entusiastas da mídia participativa por se concentrarem nas manifestações consideradas mais de "vanguarda artística” ou pós-modernas do "cool” - mashups criadas por fãs ou crítica política contracultural -, enquanto negligenciavam as práticas domésticas relativamente banais da maioria. Nossa pesquisa sugere que grande parte da produção de vídeos amadores, por exemplo, continua a ser dominada por aquilo que Richard Chalfen ${ }^{27}$ chama de "modo caseiro" - filmes domésticos sobre a vida familiar, festas de aniversário das crianças ou férias na praia. Esse material, raramente editado ou compartilhado, é mantido como um registro para ser visto em algum momento no futuro, mesmo que isso raramente aconteça. Enquanto o vídeo caseiro serve a funções determinadas em termos de memória e relações familiares, as pessoas não costumam atribuir-lhe qualquer conexão com o que assistem nos meios de comunicação predominantes: ele fica, portanto, muito longe da democratização radical da comunicação mediada, proclamada por alguns entusiastas ${ }^{28}$.

A pesquisa também sugere que há diferenças sociais marcantes relacionadas aos níveis de participação junto a essas mídias. Lenhart et al. ${ }^{29}$ sugerem que as jovens mulheres estão à frente em áreas como blogging, enquanto os homens jovens tendem a predominar no compartilhamento de vídeos. Sugerem ainda, claramente, que adolescentes provenientes de famílias suburbanas de alta renda são os que mais provavelmente editam ou compartilham on-line. Hargittai

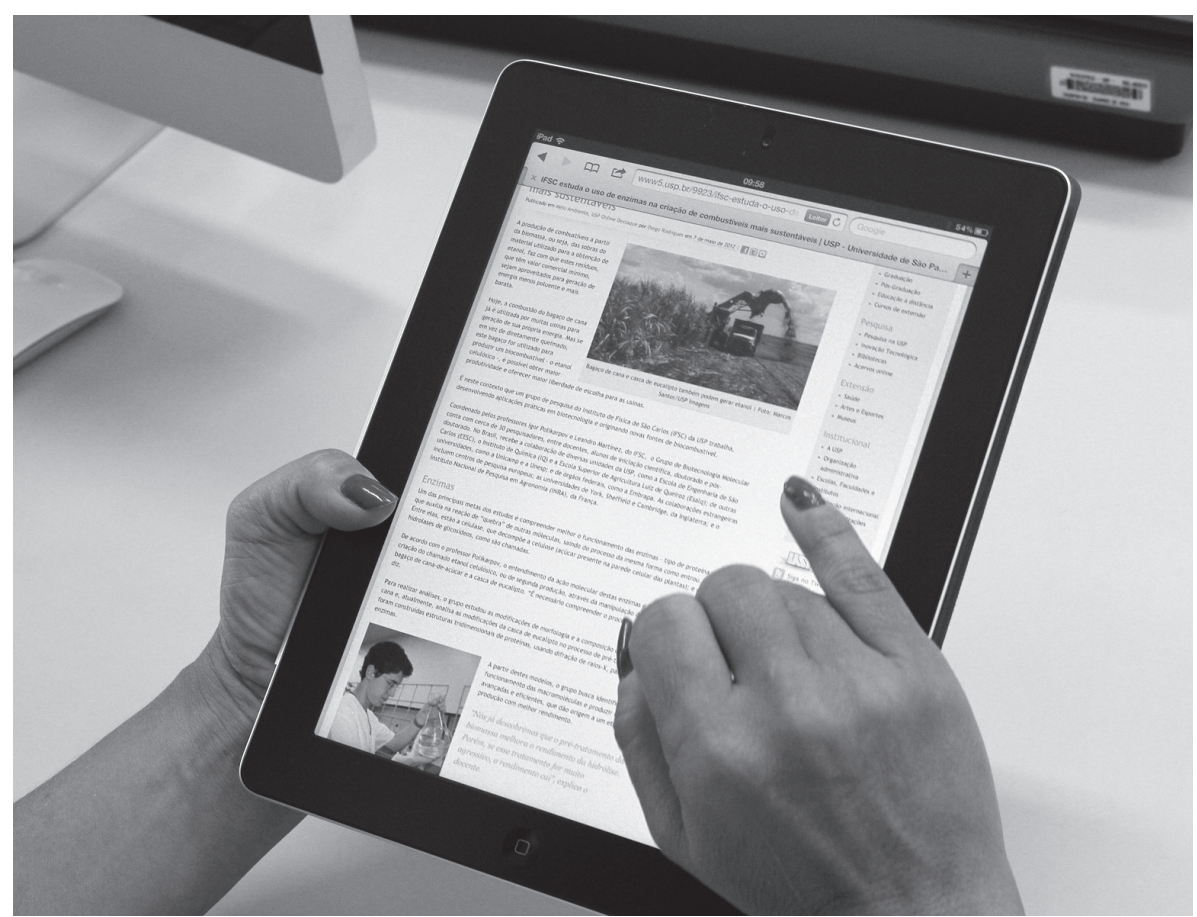

eram novas]. New York: Oxford University Press, 1988; WINSTON, Brian. Media, technology and society: a history [Mídia, tecnologia e sociedade: uma história]. London: Routledge, 1998.

22. EISENSTEIN, Elizabeth. The printing press as an agent of change [A imprensa como um agente de mudança]. New York: Cambridge University Press, 1979.

23. LENHART, Amanda; MADDEN, Mary; MACGILL, Rankin; SMITH, Aaron. Teens and social media [Os adolescentes e a mídia social]. Washington: Pew Internet and American Life Project, 2007.

24. AUCHARD, Eric. Participation on Web 2.0 sites remains weak [A participação nos sites da Web 2.0 permanece fraca]. Disponível em: <http://www.reuters. com/article/2007/04/18/ us-web-participation-id USN1743638820070418>. Acesso em: 3 dez. 2007. 25. SEFTON-GREEN, Julian; BUCKINGHAM, David. Digital visions: young people's "creative" uses of multimedia technologies in the home [Visões digitais: os usos "criativos" das tecnologias de multimídia pelos jovens em casa]. Convergence, 2, p. 47-79, 1996.

26. BURGESS, Jean. Hearing ordinary voices: Cultural Studies, vernacular creativity and digital storytelling [Ouvindo vozes comuns: estudos culturais, criatividade vernacular e narração digital de histórias]. Continuum, 20, p. 201-214, 2006.

27. CHALFEN, Richard. Snapshot versions of life [Versões da vida em instantâneos]. Bowling Green: Bowling Green State University Press, 1987. 
comunicação \& educação • Ano XVII • número 2 • jul/dez 2012

28. BUCKINGHAM, David WILLETT, Rebekah (eds.). Video cultures: Media technology and amateur creativity [As culturas do vídeo: tecnologia de mídia e criatividade amadora]. Basingstoke: Palgrave 2009; BUCKINGHAM, David; PINI, Maria; WILLETT, Rebekah. Home truths? Video production and domestic life [Verdades caseiras? A produção de vídeo e a vida familiar]. Ann Arbor: University of Michigan Press (no prelo)

29. LENHART, Amanda MADDEN Mary: MAC GILL, Rankin; SMITH, Aaron. Teens and social media [Os adolescentes e a mídia social]. Washington: Pew Internet and American Life Project, 2007.

30. HARGITTAI, Eszter WALEJKO Gina. The participation divide: content creation and sharing and the digital age $[\mathrm{O}$ diviso da participação: criação de conteúdo e compartiIhamento e a era digital]. Information, Communication and Society, 11, p. $239-256,2008$

31. WARSCHAUER, Mark Technology and social inclusion: Rethinking the digital divide [Tecnologia e inclusão social: Repensando o divisor digital]. Cambridge: MIT Press, 2003

32. MURDOCH, Rupert. His space [Seu espaço]. Entrevista Spencer Reiss. Disponível em: <www. wired.com/wired/archive/14.07/murdoch.html>. Acesso em: 12 abr. 2007

33. PETERSEN, Soren Mork. Loser generated content: from participation to exploitation Conteúdo gerado por perdedores: da participação à exploração]. First Monday 13(3). Disponivel em: <http://www.uic edu/htbin/cgiwrap/bin/ ojs/index.php/fm/issue/ view/263/showToc $>$. Acesso em: 20 ago. 2008. e Walejko ${ }^{30}$ também apontam um desequilíbrio de classes sociais - enquanto Warschauer $^{31}$, em estudo mais antigo, afirma que, ao mesmo tempo que as pessoas em comunidades menos favorecidas podem, cada vez mais, possuir computadores, há também menos probabilidade de possuir as capacitações de multimídia e banda larga necessárias à criação e compartilhamento de conteúdos mais sofisticados.

Como está implícito, "divisores digitais" ainda são aparentes, ao mesmo tempo que precisamos diferenciar os tipos de conteúdo e acesso, considerando o conhecimento técnico ou as habilidades (formas de capital tecnológico, cultural ou educacional) que estão em jogo. As desigualdades em níveis de participação estão claramente relacionadas a formas mais amplas de desigualdade social - coincidindo, em grande parte, com outras diferenças, a exemplo de como famílias de distintas classes sociais usam as dimensões educacionais da internet ou participam de atividades criativas ou artísticas quando estão desconectadas. Em sua maioria, os participantes mais ativos no mundo criativo da Web 2.0 são os "mesmos de sempre". Na verdade, se a participação on-line for tão importante social, cultural e politicamente quanto os entusiastas sugerem, então parece provável que, longe de acabar com a desigualdade social, ela pode na verdade vir a acentuá-la.

Finalmente, há a questão dos interesses comerciais em jogo nesses casos. Uma das características paradoxais da ideologia californiana é a atração que exerce, igualmente, sobre radicais políticos libertários e sobre os atuais gurus de negócios. O magnata das comunicações Rupert Murdoch ${ }^{32}$, por exemplo, expressa sua visão igualitária do futuro das comunicações: "A tecnologia está tirando o poder dos editores, das casas de publicações, dos grupos dominantes da sociedade, da elite da mídia... agora é o público que está no controle".

De fato, a explosão aparente da participação democrática na mídia está sendo acompanhada por uma crescente concentração de poder nas mãos de um pequeno número de empresas globais. A economia política da Web 2.0 ainda está se desenvolvendo - à época deste escrito, por exemplo, o site YouTube ainda não gerou lucro, apesar de ser o segundo mais visitado via on-line. Mesmo assim, a internet é um meio de comunicação excepcionalmente eficiente para atingir nichos de mercado, especialmente por seu potencial de direcionar e vigiar os consumidores individuais. De fato, grande parte desse marketing é, ele próprio, "gerado pelo usuário" e "interativo" (como no caso do anúncio viral).

Estas questões também se aplicam ao que Soren Petersen ${ }^{33}$ acertadamente chamou de "conteúdo gerado pelo perdedor". Muito trabalho não remunerado contribui para a produção de blogs, por exemplo, enquanto a maioria da renda permanece com as grandes corporações. No caso da rede social, os participantes tipicamente gastam um tempo enorme montando seus perfis e construindo redes - portanto, acumulando capital social e cultural - que não poderão levar consigo, se quiserem migrar para outro site. $\mathrm{O}$ que produzem torna-se, na realidade, informação pertencente à companhia - uma questão que veio à 
tona recentemente em disputas legais sobre a propriedade de direitos autorais de imagens no site Flickr da Yahoo ${ }^{34}$.

Há, evidentemente, um debate a ser discutido sobre as ramificações sociais e políticas maiores da Mídia 2.0. Pode-se argumentar que, longe de ter precipitado uma revolução democrática nas comunicações, estas novas mídias são simplesmente uma pequena parte de movimentos muito mais amplos em direção à individualização, autovigilância e autopromoção - características de como as identidades se formam e atuam nas sociedades neoliberais de consumo. Tais discussões, porém, estão fora do escopo deste capítulo: minha intenção é meramente indicar algumas das possíveis limitações e críticas desses tão celebrados desenvolvimentos. Apesar das aclamações de alguns "evangelistas" das novas mídias, os meios digitais provavelmente não resultarão em uma sociedade de produtores de mídia criativa, do mesmo modo que a imprensa não resultou em uma sociedade de autores publicados. Embora exista, certamente, uma promessa democrática nisto tudo, será preciso bem mais do que apenas tecnologia para cumpri-la.

\section{PARA ALÉM DA CELEBRAÇÃO}

Com esta visão bem mais cética do ambiente contemporâneo da mídia, como poderíamos avaliar as implicações para a educação e, especialmente, para a Educação para os Meios? Mais uma vez, quero prevenir contra alguns dos relatos mais entusiasmados que tipicamente circulam. A ideologia californiana tem suas próprias manifestações na educação, dentro da qual muitos acreditam que a tecnologia esteja transformando o aprendizado, mudando as relações de poder nas salas de aula e criando aprendizes autônomos e liberados. Essa forma de utopia cibernética está tipicamente alinhada a uma gama de conceitos muito em moda, porém mal definidos, dentre os quais "criatividade", "aprendizagem informal" e, mais recentemente, "personalização", são os mais conhecidos ${ }^{35}$. Essa retórica é fortemente promovida pelas empresas de tecnologia comercial, mas é também adotada por governos em sua tentativa de identificar uma "solução tecnológica" para o que consideram problemas da educação pública. Em tais discussões, é difícil estabelecer a diferença entre as afirmações exageradamente entusiasmadas dos formuladores de políticas educacionais (e alguns acadêmicos) e os argumentos de venda das companhias de computadores ${ }^{36}$.

Para alguns teóricos da educação aparentemente "progressistas", o ensino impulsionado pela tecnologia é, de certa forma, a prova justificadora das teorias de aprendizado centradas nas crianças nas décadas de 1960 e 1970 - embora outros afirmem que a tecnologia digital tornou a escola redundante e que o aprendizado real está acontecendo, agora, nos engajamentos "informais" das crianças com jogos ou com redes sociais on-line. Este argumento celebratório apresenta, de maneira típica, uma posição totalmente positiva e despojada de criticismo em relação aos usos populares da tecnologia. Aqueles que exaltam os benefícios dos jogos de computador para a aprendizagem tendem, por exemplo,
34. Yahoo user sues Virgin [Usuário do Yahoo processa Virgin]. Disponível em: <http://thoughtsfordeletion.blogspot. com/2007/09/flickr-user-sues-virgin.html>. Acesso em: 17 mar. 2009.

35. BUCKINGHAM, David. Beyond technology: Children's learning in the age of digital culture [Além da tecnologia: o aprendizado das crianças na era da cultura digital]. Cambridge: Polity, 2007.

36. Id. 
comunicação \& educação • Ano XVII • número 2 • jul/dez 2012

37. GEE, James Paul. What video games have to teach us about learning and literacy $[\mathrm{O}$ que os videogames têm para nos ensinar sobre aprendizado e literacia]. Basingstoke: Palgrave, 2003.

38. COHEN, Phil. Really useful knowledge [Conhecimento realmente útil]. Stoke-on-Trent: Trentham, 1990.

39. ROSS, Andrew. No-collar: The humane workplace and its hidden costs [Menos categorizados: o local de trabalho humano e seus custos escondidos]. Philadelphia: Temple University Press, 2003.

40. VAN DIJK, Jose. Users like you? Theorizing agency in user-generated content [Usuários como você? Teorizando a agência no conteúdo gerado pelo usuário]. Media, Culture \& Society, 31(1), p. 41-58, 2009. a ignorar as dimensões comerciais desses jogos, preferindo evitar questões embaraçosas sobre seus valores e ideologias (por exemplo, Gee, 2003) ${ }^{37}$. Envolvem-se, ainda, na valorização mal definida da "aprendizagem informal", considerando a aprendizagem formal como algo inerentemente ruim.

Outro modo de celebração particularmente presente no afeito à Educação para os Meios diz respeito à "vocação”. O currículo para os jovens de 14 a 19 anos no Reino Unido, nas últimas décadas, tem sofrido uma série aparentemente infindável de inovações que parecem programadas para abordar o problema do trabalho em uma "economia do conhecimento" pós-industrial. No caso da mídia, há sempre a esperança de que os jovens provindos de ambientes economicamente desfavorecidos sejam capazes, de alguma forma, de "tirar vantagem" do seu capital cultural, usando cursos profissionalizantes de mídia como uma maneira de evidenciar os elementos da cultura popular e que lhes permitiria conseguir um emprego ${ }^{38}$. A mais recente inovação desse tipo no Reino Unido é uma série de novos diplomas - qualificações que pretendem cruzar a divisão acadêmico-vocacional e preparar estudantes para novos tipos de situações de trabalho. Esses cursos, atualmente, recebem abundantes fundos do governo, embora seus professores estejam penando para conseguir lidar com as novas estruturas curriculares e os novos modos de avaliação - para não mencionar o excesso de novos jargões. Os cursos que vi até agora estão bem alinhados com a "Mídia 2.0": capacitam os alunos a montarem websites, programarem eventos no ambiente Second Life e criarem fotoblogs e machinima. Contudo, o fato é que tais cursos, provavelmente, serão considerados caminhos alternativos para estudantes definidos como "pessoas com baixo desempenho": ou seja, não substituirão os exames já existentes, de alto nível, que continuarão sendo valorizados pelas melhores universidades.

Os novos cursos, no entanto, oferecem uma promessa sedutora de emprego aos jovens, em ocupações consideradas interessantes e modernas, desempenhando trabalhos menos categorizados nas indústrias culturais e de tecnologia, como Andrew Ross $^{39}$ descreve. Ainda assim, apesar da mitologia, há bem poucos exemplos de fãs ou entusiastas amadores que atravessam a linha do trabalho não pago para um trabalho pago - e, como Ross explica, na indústria de jogos de computador ou nas companhias de internet, as condições de trabalho para os jovens são, muitas vezes, marcadas pela forte exploração. De fato, é de muito interesse para as indústrias de mídia ter um grande grupo de fãs desejando se tornar profissionais, especialmente porque isso lhes permite considerável liberdade para explorar os seus trabalhadores ${ }^{40}$.

\section{LIDANDO COM AS DIVISÕES DIGITAIS}

Em lugar de reduzir a escolarização a uma mera irrelevância, ou mesmo a uma simples plataforma de treinamento para a nova "economia digital", eu diria que o advento da tecnologia digital indica uma necessidade de se estender 
a missão tradicional da escola como uma instituição pública. Em colaboração com outras instituições públicas, a escola existe, em parte, para proporcionar aos jovens experiências sociais, intelectuais e culturais que eles não teriam sem ela. Evidentemente, as escolas sempre tiveram, também, funções específicas em termos de regulação das populações e de reprodução das relações sociais. No entanto, existe uma retórica modernista poderosa sobre a escola como um elemento da esfera pública que precisa ser abordada aqui.

No contexto das contínuas divisões digitais, as escolas deveriam desempenhar um papel fundamental na tentativa de nivelar as desigualdades de participação. Como Henry Jenkins e outros argumentam, as escolas têm de abordar o "hiato de participação", ou seja, o acesso desigual às oportunidades, experiências, habilidades e conhecimentos que irão preparar a juventude para uma ampla participação no mundo de amanhã" ${ }^{41}$ Assim como Jenkins, vejo isso de forma mais ampla do que apenas o acesso a equipamentos: trata-se de competências culturais, habilidades sociais e conhecimento - desdobrando-se em uma lista extensa que, como também ele citou, inclui habilidades relacionadas a jogos, experimentação e solução de problemas; capacidade de manusear diferentes fontes de mídia e modos de comunicação, navegando neles e entre eles; habilidade em fazer networking e colaborar, localizar informações e interagir com os outros; e habilidades relacionadas à avaliação crítica e capacidade de julgamento.

Enquanto algumas das habilidades identificadas por Jenkins e outros são certamente novas, outras são definitivamente tradicionais. Isto foi confirmado, por exemplo, por meio de uma pesquisa que estou realizando atualmente com colegas sobre o papel da internet na promoção da participação cívica (ver www.civicweb.eu). Enquanto alguns consideram a internet como um meio de reengajar jovens que atualmente mostram desinteresse e descontentamento em relação às organizações cívicas ou políticas, nós verificamos que tais oportunidades estão sendo abraçadas, em grande parte, pelos "mesmos de sempre": os que já têm um interesse estabelecido por questões sociais/políticas e motivação para se engajarem no debate. Nesse sentido, é possível que a internet venha a acentuar as desigualdades existentes em vez de ajudar a superá-las. Jovens desengajados e insatisfeitos - ou menos favorecidos - que queiram participar precisam desenvolver habilidades relativamente tradicionais de localização e avaliação de informações, a fim de construir argumentos e pensar criticamente; essas habilidades, por sua vez, dependem da capacidade razoavelmente avançada de leitura e escrita tradicionais.

Isso não implica, no entanto, dizer que nada está mudando. A internet talvez possa estar estimulando novas formas e estilos de engajamento cívico, no mínimo, potencialmente mais inclusivos. No entanto, nesta área como em muitas outras, a participação também requer formas relativamente tradicionais de capital cultural e educacional. Portanto, a abordagem do "hiato de participação" depende da discussão mais ampla das desigualdades: este hiato não desaparecerá com a simples ampliação do acesso à tecnologia.
41. JENKINS, Henry; CLINTON, Katie; PURUSHOTMA, Ravi; ROBISON, Alice J.; J. e WEIGEL, Margaret. Confronting the challenges of participatory culture: Media education for the $21^{\text {st }}$ century [Confrontando os desafios da cultura participativa: Educação para os Meios para o século XXI]. Disponível em: <http://www.digitallearning.macfound.org $>$. Acesso em: 27 nov. 2007. 
comunicação \& educação • Ano XVII • número 2 • jul/dez 2012

\section{O LUGAR DA CRÍTICA}

Os tipos de aprendizagem tipicamente celebrados em discussões sobre a tecnologia digital na educação tendem a oferecer pouco espaço para a reflexão crítica ou o desenvolvimento explícito das habilidades de crítica. Parece haver a presunção de que a participação ou produção criativa é algo intrinsecamente bom - e de que ela ou equivale ou gera automaticamente um entendimento crítico por conta própria. De fato, como já vimos, Gauntlett ${ }^{42}$ expressa uma forte suspeita a respeito da crítica, como se ela representasse realmente uma imposição "condescendente" da autoridade pedagógica. Ele argumenta que as audiências das mídias "já são intérpretes extremamente capazes dos conteúdos de mídia”: elas não precisam aprender a ser críticas.

Como já discuti em outros artigos (por exemplo, em Buckingham, 200343), existe certa justificação na atitude de suspeitar da crítica. O adjetivo "crítico" é um termo frequentemente relacionado à divisão entre "nós e eles" - ou seja, as pessoas qualificadas como críticas são muitas vezes, simplesmente, aquelas que concordam conosco, ao passo que as que discordam de nós são tidas como aquelas que não sabem criticar. Isto representa uma versão do que foi chamado de "efeito terceira pessoa" em discussões sobre a influência das mídias ${ }^{44}$ : sempre os outros são considerados como não tendo competência crítica. Existe sempre também o perigo de que a "capacidade crítica" se torne uma das rotinas padronizadas ou um dos jogos de linguagem das aulas de mídias - um jogo no qual os alunos simplesmente devolvem ao professor as formas de discurso crítico que receberam dele. A ênfase na análise crítica pode sancionar uma abordagem racionalista bastante superficial a respeito da mídia e, até mesmo, uma forma de cinismo superficial incapaz de representar fielmente as maneiras complexas (e

42. GAUNTLETT, David. Wide angle: is it time for Media Studies 2.0? [Grande angular: é chegada a hora para os Estudos de Mídia 2.0?]. Media Education Association Newsletter, 5, 3-5, 2007.

43. BUCKINGHAM, David. Media education: Literacy, learning and contemporary culture [Educação para os Meios: Literacia, aprendizado e cultura contemporânea]. Cambridge: Polity, 2003.

44. PERLOFF, Richard. The third person effect $[\mathrm{O}$ efeito da Terceira pessoa]. In: BRYANT, Jennings; D. ZILLMANN, Dolf (eds.) Media effects: Advances in theory and research [Efeitos da mídia: avanços na teoria e pesquisa]. Mahwah, NJ: Erlbaum, p. 489-506, 2002. não menos emocionais) pelas quais nós realmente nos relacionamos com a mídia.

No entanto, nada disto implica que as audiências já sejam ou sempre sejam "extremamente" capazes e críticas, ou que saibam tudo que precisam saber. Tampouco significa que podemos jogar fora as ferramentas e perspectivas críticas que empregamos para analisar a mídia. Podemos aceitar, sim, que as audiências sejam ativas, discriminadoras e de fato "críticas" - porém, ao mesmo tempo, também reconhecendo que há aspectos que geralmente desconhecem a respeito das mídias e que, de fato, precisam saber. Há um acúmulo de conhecimento sobre como a mídia funciona, suas indústrias e sua história, bem como sobre seus usos e efeitos na sociedade. É, sem dúvida, um acúmulo de conhecimento contestado e em constante mudança - mas, ainda assim, um acúmulo de conhecimento de acordo com critérios compartilhados que determinam o que conta como sendo verdadeiro. O perigo está em supor que o foco sobre a crítica, necessariamente, resulte em um modelo de efeitos negativos, ou que a crítica de algum modo acarrete uma imposição ilegítima de autoridade, pressupondo que as "pessoas comuns" sejam estúpidas ou iludidas. Esta ideia parece, por sua vez, implicar um sentido de crítica bastante antiquado, estreito, necessariamente negativo ou, pelo menos, uma noção de crítica como mera forma de defesa ou inoculação contra influências. 
Jenkins e seus colegas ${ }^{45}$ estão corretos mais uma vez quando identificam outro "hiato", desta vez relacionado à crítica. Como sugerem, precisamos capacitar os jovens a se tornarem participantes ativos na cultura de mídia; porém, não basta apenas participação ou criatividade por si próprias. É preciso, também, que sejam participantes críticos, desenvolvendo um entendimento mais amplo das dimensões econômicas, sociais e culturais da mídia - entendimento este que não resulta automaticamente da produção criativa. Como Carmen Luke ${ }^{46}$ discute em relação a aprender a ler e escrever, os alunos não desenvolvem uma maneira crítica de ler e escrever textos somente através da própria experiência de leitura e escrita: eles têm de se afastar da experiência imediata para poderem refletir e analisar.

Isto leva à clássica e complexa questão sobre como integrar teoria (análise crítica) e prática (produção criativa). Como o aprendizado passa do domínio da "leitura" da mídia para o domínio da "escrita" e vice-versa? Como promover reflexão crítica significativa (e não superficial) sobre o que os alunos fazem como participantes ou criadores de mídia? Como podemos ajudá-los a entender essas experiências em um contexto social e cultural mais amplo?

Acredito que a tecnologia digital nos oferece novas maneiras de abordar esta questão e de aproximar a teoria da prática. Por exemplo, no caso da edição digital e manipulação de imagem, a tecnologia pode ajudar a explicitar os processos de escolha, seleção, construção e manipulação que, nas formas analógicas, parecem estar frequentemente "trancados". Enquanto os alunos arrastam e soltam fotos na linha do tempo em um programa de edição digital, a experiência de montar e remontar uma sequência e de discutir enquanto fazem isso é um grande diferencial na natureza do aprendizado: a experiência

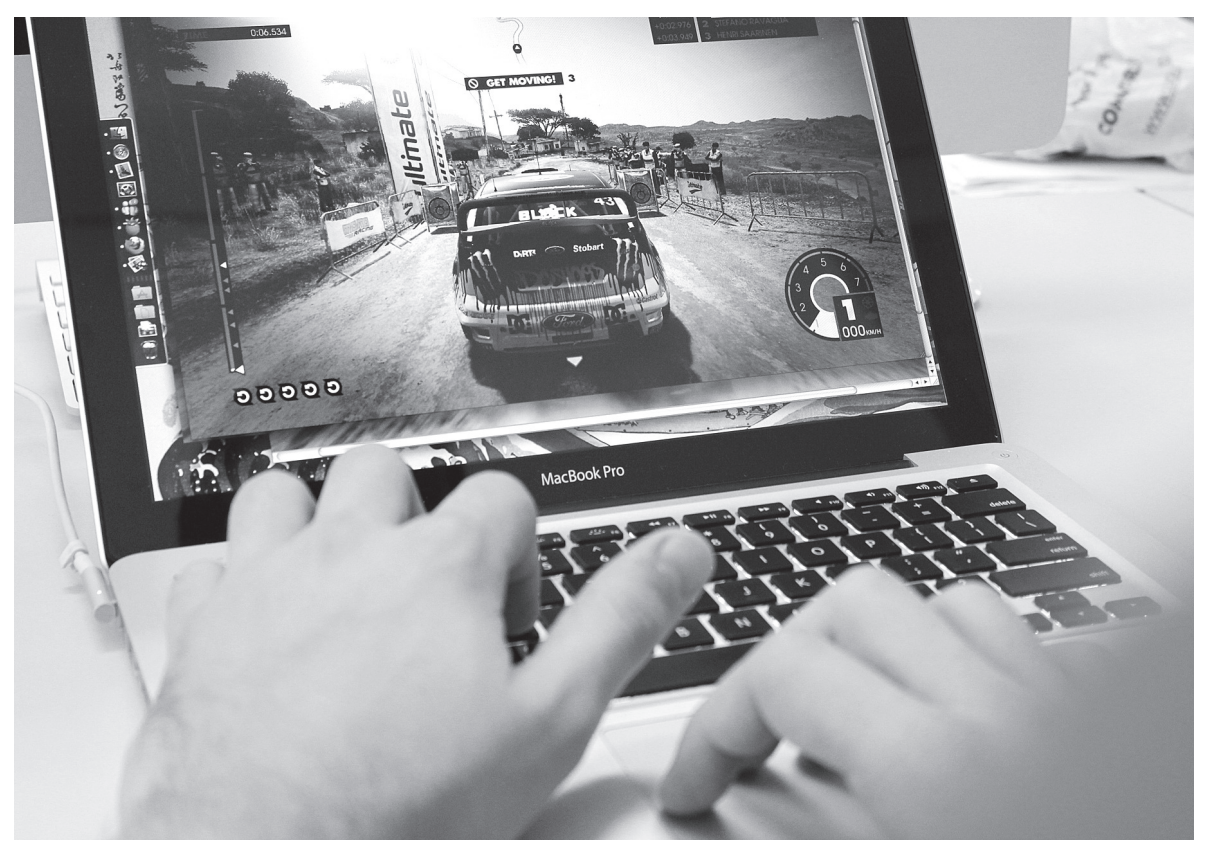

45. JENKINS, Henry; CLINTON, Katie; PURUSHOTMA, Ravi; ROBISON, Alice J.; J. e WEIGEL, Margaret. Confronting the challenges of participatory culture: Media education for the $21^{\text {st }}$ century [Confrontando os desafios da cultura participativa: Educação para os Meios para o século XXI]. Disponível em: <http://www.digitallearning.macfound.org>. Acesso em: 27 nov. 2007.

46. LUKE, Carmen. Cyber-schooling and technological change: multiliteracies for new times [A escolarização cibernética e a mudança tecnológica: multiliteracias para os novos tempos]. In: COPE, Bill; KALANTZIS, Mary (eds.). Multiliteracies: Literacy learning and the design of social futures [Multiliteracias: aprendizado da literacia e o modelo de futuros sociais]. London: Routledge, 2000. p. 69-91. 
comunicação \& educação • Ano XVII • número 2 • jul/dez 2012

47. BUCKINGHAM, David, HARVEY, Issy; SEFTON-GREEN, Julian. The difference is digital? Digital technology and student media production [A diferença é digital? A tecnologia digital e produção de mídia dos alunos]. Convergence, 5 , p. 10-20, 1999.

48. BURN, Andrew DURRAN, James. Digital anatomies: analysis as production in media education [Anatomias digitais: a análise como produção em Educação para os Meios]. In: BUCKINGHAM, David; WILLETT, Rebekah (eds.). Digital generations, cit., p. 273-294.

49. ERSTAD, Ola; GILJE, Øystein; DE LANGE, Thomas. Remixing multimodal resources: multiliteracies and digital production in Norwegian media education [Remixing recursos multimodais: multialfabetizações e produção digital em Educação para os Meios na Noruega]. Learning, Media and Technology, 27, p. 183-198, 2007.

50. DROTNER, Kirsten. Learning is hard work: digital practices and future competencies [Aprender é tarefa árdua: práticas digitais competências futuras]. In: BUCKINGHAM, David (ed.). Youth, identity and digital media [Jovens, identidade e mídia digital]. Cambridge, MA: MIT Press, 2008. p. 167-184.

51. BURN, Andrew; DUR RAN, James. Media literacy in schools [A literacia mediática nas escolas]. London: Paul Chapman 2007

52. PELletier, Caroline. Games and learning: what's the connection? [Games e aprendizado: qual é a conexão?)]. International Journal of Learning and Media 1(1), 2009. Disponível em: $<$ http://ijlm.net/>. Acesso em: 18 mar. 2009 de editar não somente é mais fácil, mas também mais explícita do que quando feita com a tecnologia analógica antiga ${ }^{47}$.

Meu colega Andrew Burn ${ }^{48}$ analisou como os professores podem usar o potencial de "remixagem" da mídia digital (uma prática característica da Mídia 2.0) para aproximar teoria e prática. As atividades já bem estabelecidas no ensino da mídia - tais como fazer um trailer para lançar, para uma nova audiência, um filme que já existe no mercado (Psicose e Romeu e Julieta são dois exemplos citados por Burn) - tornaram-se muito mais praticáveis e controláveis do que acontecia com a tecnologia analógica. Esse processo também proporciona novas oportunidades para análise e reflexão, embora (como Burn sugere) precise haver uma expectativa explícita a respeito disso já incluída dentro do processo. De fato, os casos estudados por Burn oferecem amplas provas de alunos aplicando os "não procedimentos de falsos especialistas" de semiótica e análises de economia política para desenvolver tal reflexão.

Ola Erstad e seus colegas ${ }^{49}$, assim como Kirsten Drotner ${ }^{50}$, também escreveram recentemente sobre esse remix, observando como estudantes pesquisam material na internet para seus trabalhos digitais e depois processam e recombinam esse material de várias maneiras, usando o que chamam de "capacidade de corte e cola". No entanto, esses autores também sugerem o perigo de se deixar seduzir pelo "brilho" profissional superficial desse tipo de trabalho, bem como pela aparente facilidade que os jovens têm em lidar com tecnologia. Na verdade, há muitas coisas que eles desconhecem ou não sabem como fazer - e a atividade de fazer mídia precisa ser acompanhada pela análise e conceitualização teórica, assim como por um conjunto de objetivos curriculares claros por parte dos professores.

Finalmente, nossa própria pesquisa recente sobre a criação de jogos digitais oferece mais exemplos da conexão entre teoria e prática (Buckingham e Burn, 200751; Pelletier, 200952). Trabalhamos nesse projeto com uma empresa de software educacional e um grupo de escolas para desenvolver uma ferramenta para autores de jogos: o pacote resultante, MissionMaker, possibilita aos usuários criarem jogos tridimensionais sem a necessidade de programação. Uma coisa percebida rapidamente foi que mesmo os alunos muito experientes em jogos eletrônicos não tinham, automática ou necessariamente, habilidade para criar jogos - uma atividade complexa que envolve pensamento computacional, lógica e a capacidade de imaginar um usuário que não é somente uma audiência (ou um leitor), mas sim um jogador que interage com o texto. Para poder passar da posição de jogador à posição de inventor de jogos, é necessário retroceder da experiência imediata e engajar-se em análises aprofundadas do sistema.

\section{CONCLUSÃO}

Precisamos realmente da Educação para os Meios 2.0? Talvez sim; mas, certamente, ainda precisamos também da Educação para os Meios 1.0. Os defensores dos Estudos de Mídia 2.0 identificam alguns dos imperativos em 
Precisamos realmente de educação para os meios? - David Buckingham

relação a isso. Eu não aceitaria a afirmação de Merrin $^{53}$ de que perderemos "o direito de ensinar", se não participarmos ativamente de toda a gama de mídia contemporânea. Contudo, concordo que é necessário manter-se a par das experiências de mídia de nossos alunos, bem como de suas orientações (que estão sempre mudando) em relação à mídia. Apesar disso, precisamos também tomar cuidado para não pressupor que essas experiências são todas iguais (o argumento da "geração digital"); da mesma forma, a necessidade de continuar a par das experiências de nossos alunos não significa que tenhamos de importar automaticamente os mais recentes dispositivos tecnológicos para uso em sala de aula, tampouco que precisamos começar a "incrementar" nossos perfis no Facebook em um baldado esforço para "fazer parte da galera".

As novas mídias oferecem também novas oportunidades de participação, de comunicação criativa e de geração de conteúdo, pelo menos para pessoas e em contextos específicos. No entanto, as competências necessárias para abraçar essas oportunidades não são igualmente distribuídas entre as pessoas e não surgem, simplesmente, porque elas têm acesso à tecnologia. Igualmente, é errado presumir que a participação, por si só, é sempre uma coisa positiva ou necessariamente democrática, contracultural ou emancipadora. A produção criativa pode ser um poderoso meio de aprendizagem, seja envolvendo vários tipos de remix, a apropriação e adaptação de textos existentes ou a criação de textos completamente novos - ou ainda, simplesmente, explorando o potencial para comunicação em rede. No entanto, tudo isso necessita reflexão crítica e precisa ser combinado com análise crítica, embora a busca de como aconteceria essa combinação seja verdadeiramente difícil.

Em termos mais amplos, a própria Educação para os Meios precisa adotar uma posição mais forte e crítica em relação à celebração da tecnologia na educação, bem como ao tipo de tecnofetichismo erroneamente considerado por alguns como a última palavra em mudança educacional. A Educação para os Meios corre o risco de ser vista como apenas mais um meio de importar tecnologia de computação para as escolas - ou como uma alternativa atraente em contraste com a aridez das planilhas eletrônicas, a administração de arquivos e o treinamento instrumental que constituem a maior parte dos cursos de "tecnologia da informação" nas escolas. Aqui existe uma oportunidade, mas os imperativos críticos tradicionais da Educação para os Meios - que vão muito além das habilidades práticas ou do apelo sentimental à "criatividade" - não devem ser abandonados.

\section{REFERÊNCIAS BIBLIOGRÁFICAS}

BLACKMAN, Shane. "Decanonised knowledge" and the radical project: towards an understanding of cultural studies in British universities ["Conhecimento descanonizado" e o projeto radical: para entender os estudos culturais nas universidades britânicas]. Pedagogy, Culture and Society, 8, 2000.

53. MERRIN, William. Media Studies 2.0 [Estudos de Mídia 2.0]. Disponível em: <http://twopointzeroforum.blogspot.com/>. Acesso em: 16 mar. 2009. 
comunicação \& educação • Ano XVII • número 2 • jul/dez 2012

BUCKINGHAM, David. Media education: Literacy, learning and contemporary culture [Educação para os Meios: literacia, aprendizado e cultura contemporânea]. Cambridge: Polity, 2003.

Is there a digital generation? In: BUCKINGHAM, David; WILLETT, Rebekah (eds.). Digital generations: Children, young people and new media [Gerações digitais: as crianças, os jovens e os novos meios de comunicação de massa]. Mahwah: Lawrence Erlbaum Associates, 2006.

. Beyond technology: Children's learning in the age of digital culture [Além da tecnologia: $\mathrm{O}$ aprendizado das crianças na era da cultura digital]. Cambridge: Polity, 2007.

"Creative" visual methods in media research: possibilities, problems and proposals [Os métodos visuais "criativos" na pesquisa de mídia: possibilidades, problemas e propostas]. Media, Culture and Society, 31(4), p. 633-652, 2009.

; BANAJI, Shakuntala; BURN, Andrew; CARR, Diane; CRANMER, Susan; WILLETT, Rebekah. The media literacy of children and young people: A review of the academic research [Literacia Mediática de crianças e jovens: Uma análise da pesquisa acadêmica]. Journal of Educational Media and Hypermedia, 16, p. 323-349, 2005.

; BRAGG, Sara. Media Education Goes Digital [Educação para os Meios]. Learning, Media and Technology, 27(2), (2007).

; TURNBULL, S. (eds.). Special issue: "Media Education" (Edição especial: "Educação para os Meios"), 120. Media International Australia (Austrália Mídia Internacional), 2006.

; HARVEY, Issy; SEFTON-GREEN, Julian. The difference is digital? Digital technology and student media production [A diferença é digital? A tecnologia digital e a produção de mídia dos alunos]. Convergence, 5, 1999.

; WILLETT, Rebekah (eds.). Video cultures: Media technology and amateur creativity [As culturas do vídeo: tecnologia de mídia e criatividade amadora]. Basingstoke: Palgrave, 2009.

; PINI, Maria; WILLETT, Rebekah. "Take back the tube!" The discursive construction of amateur film-and video-making ["De volta à televisão!" A construção discursiva na criação de filmes e vídeos amadores]. Journal of Media Practice, 8, 2007.

; PINI, Maria; WILLETT, Rebekah. Home truths? Video production and domestic life [Verdades caseiras? A produção de vídeo e a vida familiar]. Ann Arbor: University of Michigan Press (no prelo).

BURGESS, Jean. Hearing ordinary voices: Cultural Studies, vernacular creativity and digital storytelling [Ouvindo vozes comuns: estudos culturais, criatividade vernacular e narração digital de histórias]. Continuum, 20, 2006. 
BURN, Andrew. Repackaging the slasher movie: digital unwriting of film in the classroom [Reapresentando os filmes "slasher": um exercício de edição digital de filmes na sala de aula]. English in Australia, 127-8, p. 1-19, 2000.

; DURRAN, James. Digital anatomies: analysis as production in media education [Anatomias digitais: a análise como produção em Educação para os Meios]. In: BUCKINGHAM, David; WILLETT, Rebekah (eds.). Digital generations: Children, young people and new media [Gerações Digitais: Crianças, jovens e novos meios de comunicação de massa]. Mahwah, NJ: Erlbaum, 2006.

. Media literacy in schools [A literacia mediática nas escolas]. London: Paul Chapman, 2007.

CHALFEN, Richard. Snapshot versions of life [Versões da vida em instantâneos]. Bowling Green: Bowling Green State University Press, 1987.

COHEN, Phil. Really useful knowledge [Conhecimento realmente útil]. Stokeon-Trent: Trentham, 1990.

DROTNER, Kirsten. Learning is hard work: digital practices and future competencies [Aprender é tarefa árdua: práticas digitais e competências futuras]. In: BUCKINGHAM, David (ed.). Youth, identity and digital media [Jovens, identidade e mídia digital]. Cambridge, MA: MIT Press, 2008.

EISENSTEIN, Elizabeth. The printing press as an agent of change [A imprensa como um agente de mudança]. New York: Cambridge University Press, 1979.

ERSTAD, Ola; GILJE, Øystein; DE LANGE, Thomas. Remixing multimodal resources: multiliteracies and digital production in Norwegian media education [Remixing recursos multimodais: multialfabetizações e produção digital em comunicação para os meios na Noruega]. Learning, Media and Technology, 27, 2007.

FACER, Keri; FURLONG, Ruth. Beyond the myth of the "cyberkid": young people at the margins of the information revolution [Além do mito do "cyberkid": jovens à margem da revolução informática]. Journal of Youth Studies, 4, 2001.

GAUNTLETT, David. Wide angle: is it time for Media Studies 2.0? [Grande angular: é chegada a hora para os Estudos de Mídia 2.0?]. Media Education Association Newsletter, 5, 2007.

GEE, James Paul. What video games have to teach us about learning and literacy [O que os videogames têm para nos ensinar sobre aprendizado e literacia]. Basingstoke: Palgrave, 2003.

GROSSBERG, Lawrence. Cultural Studies vs. political economy: is anybody else bored with this debate? [Estudos culturais vs. economia política: há mais alguém entediado com esse debate?]. Critical Studies in Mass Communication, 12, 1995.

HARGITTAI, Eszter; WALEJKO, Gina. The participation divide: content creation and sharing and the digital age [O divisor da participação: criação de conteúdo e compartilhamento e a era digital]. Information, Communication and Society, 11, 2008. 
comunicação \& educação • Ano XVII • número 2 • jul/dez 2012

HERRING, Susan. Questioning the c divide: technological exoticism and adult constructions of online youth identity [Questionando o divisor geracional: exoticismo tecnológico e construções adultas da identidade on-line do jovem]. In: BUCKINGHAM, David (ed.). Youth, identity and digital media [Juventude, identidade e mídia digital]. Cambridge: MIT Press, 2008.

LENHART, Amanda; MADDEN, Mary; MACGILL, Rankin; SMITH, Aaron. Teens and social media [Os adolescentes e a mídia social]. Washington: Pew Internet and American Life Project, 2007.

LUKE, Carmen. Cyber-schooling and technological change: multiliteracies for new times [A escolarização cibernética e a mudança tecnológica: multiliteracias para os novos tempos]. In: COPE, Bill; KALANTZIS, Mary (eds.). Multiliteracies: Literacy learning and the design of social futures [Multiliteracias: aprendizado da literacia e o modelo de futuros sociais]. London: Routledge, 2000.

MARVIN, Carolyn. When old technologies were new [Na época em que as velhas tecnologias eram novas]. New York: Oxford University Press, 1988.

MATON, Karl; WRIGHT, H. K. Returning Cultural Studies to Education [Devolvendo os estudos culturais à educação]. International Journal of Cultural Studies, 5(4).

OFCOM. Media Literacy: Report on UK Children's Media Literacy [Literacia mediática: relatório sobre a Literacia Mediática das crianças do Reino Unido]. London: Ofcom, 2008.

PERLOFF, Richard. The third person effect [O efeito da terceira pessoa]. In: BRYANT, Jennings; D. ZILLMANN, Dolf (eds.). Media effects: Advances in theory and research [Efeitos da mídia: avanços na teoria e pesquisa]. Mahwah, NJ: Erlbaum, 2002.

ROBINS, Kevin; WEBSTER, Frank. Times of the technoculture [Época da tecnocultura]. London: Routledge, 1999.

ROSS, Andrew. No-collar: The humane workplace and its hidden costs [Menos categorizados: o local de trabalho humano e seus custos escondidos]. Philadelphia: Temple University Press, 2003.

SEFTON-GREEN, Julian; BUCKINGHAM, David. Digital visions: young people's "creative" uses of multimedia technologies in the home [Visões digitais: os usos "criativos" das tecnologias de multimídia pelos jovens em casa]. Convergence, 2, 1996.

STREETER, Thomas. The cable fable revisited: discourse, policy and the making of cable television [A fábula do cabo revista: discurso, política e a criação da televisão a cabo]. Critical Studies in Mass Communication, 4, p. 174-200, 1987.

VAN DIJK, Jose. Users like you? Theorizing agency in user-generated content [Usuários como você? Teorizando a agência no conteúdo gerado pelo usuário].

Media, Culture \& Society, 31(1), 2009. 
Precisamos realmente de educação para os meios? - David Buckingham

WARSCHAUER, Mark. Technology and social inclusion: Rethinking the digital divide [Tecnologia e inclusão social: Repensando o divisor digital]. Cambridge: MIT Press, 2003.

WINSTON, Brian. Media, technology and society: A history [Mídia, tecnologia e sociedade: Uma história]. London: Routledge, 1998.

WOOLGAR, Steve. Virtual society? Technology, cyberbole, reality [Sociedade virtual? Tecnologia, "cyberbole", realidade]. Oxford: Oxford University Press, 2002.

\section{Endereços eletrônicos}

ANDERSON, Nate. Tim Berners-Lee on Web 2.0: "nobody even knows what it means" [Web 2.0: "Ninguém nem mesmo sabe o que significa"]. Disponível em: Ars Technica (http://arstechnica.com/business/news/2006/09/7650.ars). Acesso em: 17 mar. 2009.

AUCHARD, Eric. Participation on Web 2.0 sites remains weak [A participação nos sites da Web 2.0 permanece fraca]. Disponível em: <http://www.reuters.com/ article/2007/04/18/us-web-participation-idUSN1743638820070418>. Acesso em: 3 dez. 2007.

BARBROOK, Richard; CAMERON, Andy. The Californian ideology [A ideologia californiana]. Disponível em: <http://www.hrc.wmin.ac.uk/theorycalifornianideology.html>. Acesso em: 8 nov. 2008.

JENKINS, Henry; CLINTON, Katie; PURUSHOTMA, Ravi; ROBISON, Alice J.; J. e WEIGEL, Margaret. Confronting the challenges of participatory culture: Media education for the $21^{\text {st }}$ century [Confrontando os desafios da cultura participativa: educação para os meios para o século XXI]. Disponível em: <http://www. digitallearning.macfound.org >. Acesso em: 27 nov. 2007.

MERRIN, Willian. Media Studies 2.0 [Estudos de Mídia 2.0]. Disponível em: $<$ http://twopointzeroforum.blogspot.com/>. Acesso em: 16 maio 2009.

MURDOCH, Rupert. His space [Seu espaço]. Entrevista Spencer Reiss. Disponível em: <www.wired.com/wired/archive/14.07/murdoch.html>. Acesso em: 12 abr. 2007.

O'REILLY, Tim. (2005). What is Web 2.0? Design patterns and business models for the next generation of software [O que é Web 2.0? Padrões de design e modelos de negócios para a próxima geração de software]. 2005. Disponível em: <http:// www.oreillynet.com/pub/a/oreilly/tim/news/2005/09/30/what-is-web-20. html>. Acesso em: 17 mar. 2009.

PELLETIER, Caroline. Games and learning: what's the connection? [Games e aprendizado: qual é a conexão?)]. International Journal of Learning and Media 1(1), 2009. Disponível em: <http://ijlm.net/>. Acesso em: 18 mar. 2009. 
comunicação \& educação • Ano XVII • número 2 • jul/dez 2012

PETERSEN, Soren Mork. Loser generated content: from participation to exploitation [Conteúdo gerado por perdedores: da participação à exploração]. First Monday 13(3). Disponível em: <http://www.uic.edu/htbin/cgiwrap/bin/ ojs/index.php/fm/issue/view/263/showToc>. Acesso em: 20 ago. 2008.

YAHOO USER SUES VIRGIN [Usuário do Yahoo processa Virgin]. Disponível em: <http://thoughtsfordeletion.blogspot.com/2007/09/flickr-user-sues-virgin. html>. Acesso em: 17 mar. 2009.

ZIMMER, Michael. Critical Perspectives on Web 2.0 [Perspectivas críticas na Web 2.0] Disponível em: <http://www.uic.edu/htbin/cgiwrap/bin/ojs/index.php/ $\mathrm{fm} /$ issue/view/263/showToc $>$. Acesso em: 20 ago. 2008. 